\title{
High Expression of Interleukin-12A and Its Association with the Clinicopathology and Prognosis of Differentiated Thyroid Cancer
}

\author{
Guo-Qiang Zhang Chen-Tian Shen Hong-Jun Song Zhong-Ling Qiu \\ Quan-Yong Luo \\ Department of Nuclear Medicine, Shanghai Jiao Tong University Affiliated Sixth People's Hospital, Shanghai, China
}

\section{Keywords \\ Differentiated thyroid cancer · Interleukin-12A · Prognosis}

\begin{abstract}
Background: The inflammatory microenvironment is closely related to the occurrence and development of cancer. Members of the interleukin-12 (IL-12) cytokine family play synergistic or antagonistic roles in the tumor microenvironment, in the form of classic heterodimers or newly discovered monomers or homodimers. Objective: The purpose of this study was to investigate the association between IL-12A and the clinicopathology and prognosis of differentiated thyroid cancer (DTC). Methods: A total of 101 pathologically confirmed DTC patients were included in this study. Immunohistochemistry was performed to assess IL-12A expression in DTC and corresponding paracancerous tissues. The associations of IL-12A with clinicopathology and prognosis were evaluated. Results: IL-12A was expressed in both normal thyroid tissues and DTC, but its expression level was significantly higher in DTC than in normal thyroid tissues $(p<$ $0.001)$. IL-12A was positively correlated with tumor size ( $p=$ 0.027 ), risk stratification ( $p=0.020)$, and TNM (Tumor-NodeMetastasis) stage ( $p=0.024)$, but not with age, sex, patho-
\end{abstract}

karger@karger.com www.karger.com/etj

(c) 2020 European Thyroid Association Published by S. Karger AG, Basel logical type, multifocality, extrathyroid extension, lymph node metastasis, and distant metastasis (all $p>0.05$ ). Lymphocytic thyroiditis was found in 26/101 patients (25.7\%), which was negatively associated with IL-12A expression ( $p=$ 0.018). Multivariate logistic regression analysis showed that risk stratification was the significant independent predictor of IL-12A expression. The rate of disease persistence or recurrence (P\&R) was 13/101 (12.9\%), and a positive relationship was found between IL-12A expression and P\&R $(p=0.020)$. Disease-free survival was affected by factors such as tumor size, extrathyroid extension, tumor stage ( $T$ stage), and IL$12 \mathrm{~A}$ expression, with $p$ values of $0.006,0.048,0.002$, and 0.012 , respectively. Multivariate Cox proportional-hazards analysis showed that tumor size $\geq 2 \mathrm{~cm}$ (hazard ratio [HR] = 4.041 [95\% Cl: 1.144-14.274], $p=0.031$ ) and high IL-12A expression ( $\mathrm{HR}=4.027$ [95\% Cl 1.014-15.994], $p=0.049$ ) were independent predictors of prognosis of DTC patients. Conclusions: IL-12A is highly expressed in DTC and is associated with disease aggressiveness. In addition, IL-12A is an independent predictor of the outcome of DTC.

(C) 2020 European Thyroid Association Published by S. Karger AG, Basel

G.-Q. Zhang and C.-T. Shen contributed equally to this work. 


\section{Introduction}

Thyroid cancer accounts for $1 \%$ of all human tumors and is the most common malignancy of the endocrine system [1]. In the past few decades, the incidence of this disease has sharply increased as a result of environmental factors and the rapid advances in diagnostic radiology tools [2]. Thyroid cancer is characterized by different clinicopathological properties, and differentiated thyroid cancer (DTC) accounts for more than 90\% of thyroid cancer cases [3]. Although patients with DTC have a relatively good prognosis with standard treatments, $10-30 \%$ of patients suffer from persistence or recurrence (P\&R), leading to high mortality rates [4].

A close relationship between inflammation and cancer was suggested by Virchow in 1863 [5]. Since then, the association between tumor formation and the inflammatory microenvironment has been repeatedly reported [6]. The tumor microenvironment of DTC is composed of a mixture of stromal cells, immune cells, and their secreted cytokines [7]. Furthermore, the components and structures of cytokines vary greatly, resulting in synergistic and antagonistic impacts of cytokines on the occurrence and development of cancer. For example, the proliferation and invasion of thyroid cancer are promoted by (C$\mathrm{X}-\mathrm{C}$ motif) ligand 10 , but inhibited by (C-X-C motif) ligands 9 and 11 [8]. A large number of secreted proteins that bind to specific receptors and play a role in communication between leukocytes are referred to as interleukins (ILs) [9]. These cytokines are classified into different families according to sequence homology, receptor chain similarity, or functional characteristics [7]. The IL-12 family is a group of heterodimeric cytokines which consist of an $\alpha$-chain (P19, P28, or P35) and a $\beta$-chain (P40 or Ebi3). The discovery of this cytokine family spans more than 50 years [10]. The IL- 12 family includes IL-12, $-23,-27$, and -35 , all of which are composed of a specific combination of an $\alpha$-chain and a $\beta$-chain [11].

The roles of the IL-12 cytokine family are not fully understood. Researchers have focused on the antitumor effect of IL-12 or on the impacts of IL-12A (P35) and IL-12B (P40) gene polymorphisms on the progression of cancer [12-14]. The role of the IL-12 family on thyroid cancer was described in only one study, which reported a decrease in serum IL-12 expression in thyroid cancer compared with that in healthy controls; however, IL-12 expression was significantly higher in patients with active disease than in those who were disease free [15]. Few studies have reported an association between the IL-12 family and the prognosis of patients with cancer. There have been reports demonstrating that the subunits of this cytokine family could exist and function as monomers and homodimers [16]. Therefore, the purpose of this study was to examine whether IL-12A is associated with clinicopathological features and whether this cytokine could act as a marker to predict the outcome of patients with DTC.

\section{Subjects and Methods}

\section{Patients and Follow-Up}

The present study was carried out at Shanghai Jiao Tong University Affiliated Sixth People's Hospital, a major ${ }^{131}$ I treatment center in China. A total of 101 DTC patients were enrolled in the study according to the following inclusion criteria: (1) the patient had undergone total thyroidectomy, cervical lymph node dissection, and postoperative radioiodine treatment at our hospital between 2014 and 2015; (2) there was histopathological confirmation of papillary thyroid cancer or follicular thyroid cancer; (3) there was no history of other tumors and severe chronic diseases that affected survival, such as acquired immunodeficiency syndrome or kidney failure; and (4) the patient was followed up regularly. The standard follow-up protocol, which included free triiodothyronine, free thyroxine, thyroid-stimulating hormone, thyroglobulin (Tg), anti-Tg antibody, neck ultrasonography (US), chest computed tomography (CT) scans, and ${ }^{131} \mathrm{I}$ whole-body scans $\left({ }^{131} \mathrm{I}\right.$ WBS), was routinely performed.

\section{Criteria for P\&R of DTC}

A patient was considered disease free if there was no imaging evidence by neck US and chest CT after surgery and by ${ }^{131} \mathrm{I}$-WBS after radioiodine treatment, and if there was no serum evidence $\left(\mathrm{Tg}<0.2 \mathrm{ng} / \mathrm{mL}\right.$ ) during the follow-up period after ${ }^{131} \mathrm{I}$ treatment. Active disease was defined as P\&R of DTC in one of the following situations: (1) distant lesions were found and histopathologically confirmed as metastases of DTC during primary surgery; (2) distant lesions were detected by CT, were also positive in ${ }^{131} \mathrm{I}-\mathrm{WBS}$, and were accompanied by elevated serum stimulated Tg; (3) distant lesions were diagnosed by CT but were negative on ${ }^{131} \mathrm{I}-\mathrm{WBS}$, and were finally identified by fine needle aspiration biopsy or surgery; and (4) lesions were detected by US and accompanied by elevated serum Tg during follow-up, and were finally confirmed by fine needle aspiration biopsy or surgery. Disease-free survival (DFS) was defined as the time from the date of surgery to the diagnosis of distant metastasis or recurrence or to the date of the last follow-up. DFS was considered to be 0 when distant metastasis was present at the time of surgery [17].

\section{Immunohistochemical Staining and Analysis}

The most representative samples of 101 thyroid cancer and corresponding paracancerous tissues were selected to create a set of tissue microarrays with formalin-fixed, paraffin-embedded specimens, and the tissues at the same coordinate point of these microarrays in different slides were from the same patients. Immunohistochemical staining was performed on the tissue microarrays with a primary antibody against IL-12A (1:100 dilution, ab131039; Abcam) and was incubated overnight at $4{ }^{\circ} \mathrm{C}$. The specimens were then incubated with horseradish peroxidase-conjugated goat anti- 
rabbit secondary antibodies after having been rinsed with phosphate-buffered saline 3 times for 5 min each time. After 1-h incubation at room temperature, the slides were washed again with phosphate-buffered saline and were stained with diaminobenzidine; tap water was used to stop the staining process.

The dehydrated sections were restained with hematoxylin. Immunohistochemical staining was performed by two pathologists who were blinded to the patients' clinical data, each with a tissue microarray. The two stained slides were scanned with an automated digital slide scanner (Pannoramic MIDI 3DHISTECH) and were analyzed with the supporting analysis software QuantCenter. All the cells in the specimen were counted for positive rates, providing two positive rate reports for the specimens from the same patient. The final positive rate was the mean of the two. Low and high IL-12A expression was defined by the optimal positive rate cutoff value $(70.7 \%)$. The threshold of $70.7 \%$ was defined by the X-tile software (version 3.6.1; Yale University, New Haven, CT, USA), which constructs a two-dimensional projection of each possible subgroup to show the robustness of the relationship between IL-12A and outcome, as previously described [18].

\section{Statistical Analysis}

Statistical analysis was performed using SPSS Statistics 21.0 (SPSS Inc., Chicago, IL, USA). A $\chi^{2}$ test or Fisher's exact test was applied to evaluate the association between IL-12A expression and the clinicopathological characteristics and outcomes of DTC patients. The significant clinicopathological variables were then further analyzed with a multivariate logistic regression analysis model to define independent predictors of IL-12A expression. The DFS was compared using the Kaplan-Meier method, and the log-rank test was used to determine differences between two groups. Cox's proportional-hazards model was used to evaluate factors which affect DFS independently. The selection of prognostic factors, including in the analysis of the multivariate Cox regression model, was based on the clinical perspective, log-rank test results, and the reported factors potentially influencing survival. All tests were twosided, and a $p$ value $<0.05$ was considered statistically significant.

\section{Results}

\section{IL-12A Overexpression in DTC and Correlation with Clinicopathological Characteristics}

A total of 101 patients were retrospectively analyzed in the present study; the demographic and clinicopathological details on these patients are summarized in Table 1. An immunohistochemical assay was performed on the 101 cancer samples and the corresponding noncancerous tissues to evaluate the IL-12A expression levels in DTC. The mean rate of positivity for IL-12A was $49.0 \pm 24.6 \%$ in the cancer group and $33.5 \pm 24.1 \%$ in the normal group. A paired-samples $t$ test showed a significant difference between the two groups $(p<0.001)$.

IL-12A was overexpressed in DTC compared with normal paracancerous tissues. The rate of positivity for IL-12A in DTC ranged from 0.29 to $91.86 \%$, with a me-
Table 1. Demographics and clinicopathological features of the 101 enrolled patients with differentiated thyroid cancer

\begin{tabular}{|c|c|c|}
\hline \multicolumn{3}{|l|}{ Age at diagnosis } \\
\hline Mean \pm SD (range), years & $41.4 \pm 14.1(17-82)$ & \\
\hline$<55$ years & 77 & $76.2 \%$ \\
\hline$\geq 55$ years & 24 & $23.8 \%$ \\
\hline \multicolumn{3}{|l|}{ Sex } \\
\hline Male & 29 & $28.7 \%$ \\
\hline Female & 72 & $71.3 \%$ \\
\hline \multicolumn{3}{|l|}{ Pathology of DTC } \\
\hline FTC & 14 & $13.9 \%$ \\
\hline PTC & 87 & $86.1 \%$ \\
\hline \multicolumn{3}{|l|}{ T stage } \\
\hline $\mathrm{T} 1$ & 55 & $54.5 \%$ \\
\hline $\mathrm{T} 2$ & 12 & $11.9 \%$ \\
\hline T3 & 16 & $15.8 \%$ \\
\hline $\mathrm{T} 4$ & 18 & $17.8 \%$ \\
\hline \multicolumn{3}{|l|}{ Lymph node metastasis } \\
\hline No & 25 & $24.8 \%$ \\
\hline N1a & 34 & $33.7 \%$ \\
\hline $\mathrm{N} 1 \mathrm{~b}$ & 42 & $41.6 \%$ \\
\hline \multicolumn{3}{|l|}{ Distant metastasis } \\
\hline No & 93 & $92.1 \%$ \\
\hline Yes & 8 & $7.9 \%$ \\
\hline \multicolumn{3}{|l|}{ Risk stratification } \\
\hline Low risk & 18 & $17.8 \%$ \\
\hline Intermediate risk & 77 & $76.2 \%$ \\
\hline High risk & 6 & $5.9 \%$ \\
\hline \multicolumn{3}{|l|}{ TNM stage } \\
\hline I & 77 & $76.2 \%$ \\
\hline II & 20 & $19.8 \%$ \\
\hline III & 1 & $1.0 \%$ \\
\hline IV & 3 & $3.0 \%$ \\
\hline \multicolumn{3}{|l|}{ Lymphocytic thyroiditis } \\
\hline No & 75 & $74.3 \%$ \\
\hline Yes & 26 & $25.7 \%$ \\
\hline
\end{tabular}

SD, standard deviation; DTC, differentiated thyroid cancer; FTC, follicular thyroid cancer; PTC, papillary thyroid cancer; T stage, tumor stage; TNM stage, Tumor-Node-Metastasis stage.

dian of $53.2 \%$. The cutoff value for IL-12A positivity was defined as $70.7 \%$ using the X-tile software, based on the association of IL-12A with DFS in DTC patients, as shown in Figure 1a. Accordingly, the 101 patients were divided into two groups: a high IL-12A expression group, with a positivity rate $>70.7 \%$, and a low IL-12A expression group, with a positivity rate $<70.7 \%$. The expression levels of IL-12A in normal thyroid tissues, as well as in the low expression group and the high expression group, are shown in Figure 2.

The high IL-12A expression group consisted of 20 patients, including 7 male and 13 female patients, with a 


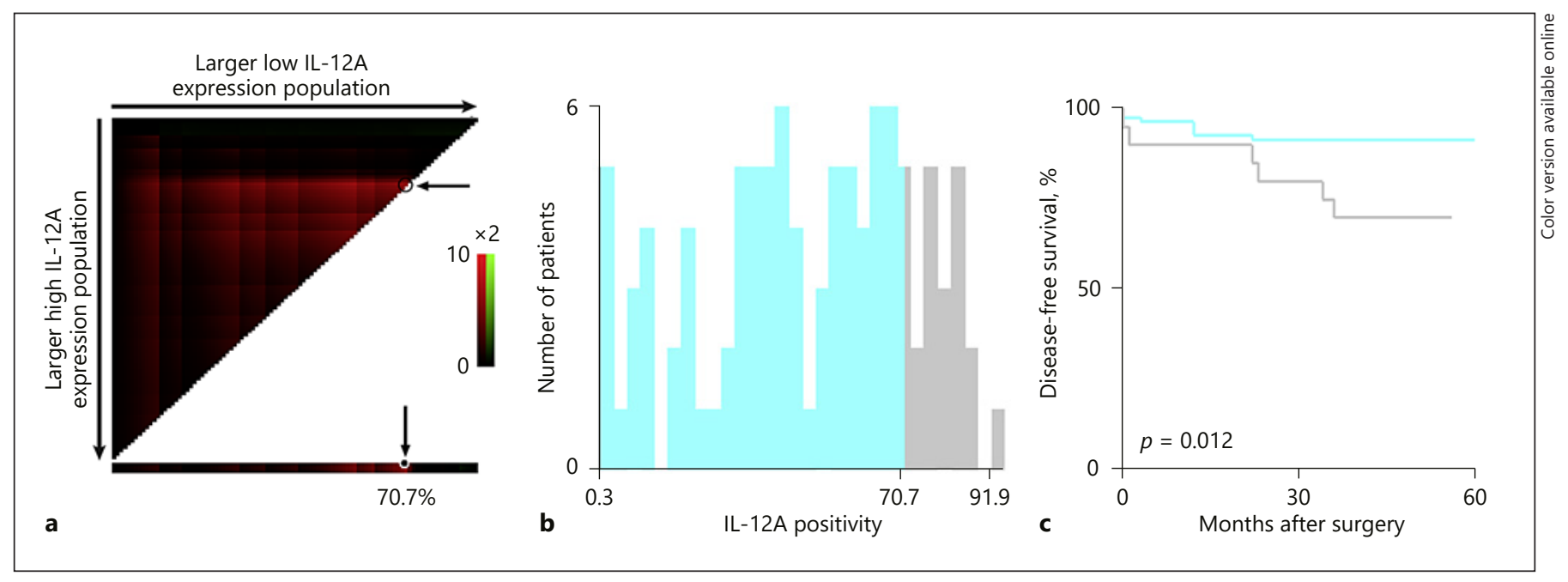

Fig. 1. X-tile analysis of survival data for differentiated thyroid cancer patients reveals a continuous distribution based on the IL-12A positivity rate. a The plot shows that a cutoff value of $70.7 \%$ was defined by the X-tile data, which is represented in a rectangulartriangular grid. The vertical axis represents all possible "high IL12 A expression" populations, with the size of the high expression population increasing from top to bottom. Similarly, the horizontal axis represents all possible "low IL-12A expression" populations, with the size of the low expression population increasing from left to right. Data along the hypotenuse represent results from a single cut-point that divides the IL-12A expression rate into low or high subsets. The strength of the inverse association between every cut-point of the IL-12A positivity rate and disease-free survival is assessed by a $\chi^{2}$ value, shown by a color code in the grid, ranging from "low" (dark, black) to "high" (bright, red). The program can select the optimal cutoff value by selecting the highest $\chi^{2}$ value, corresponding to the brightest region, marked by arrows. b The cut-point displayed on a histogram of the entire cohort. c Kaplan-Meier curve showing disease-free survival based on the IL-12A expression level (low expression subset: green; high expression subset: gray).

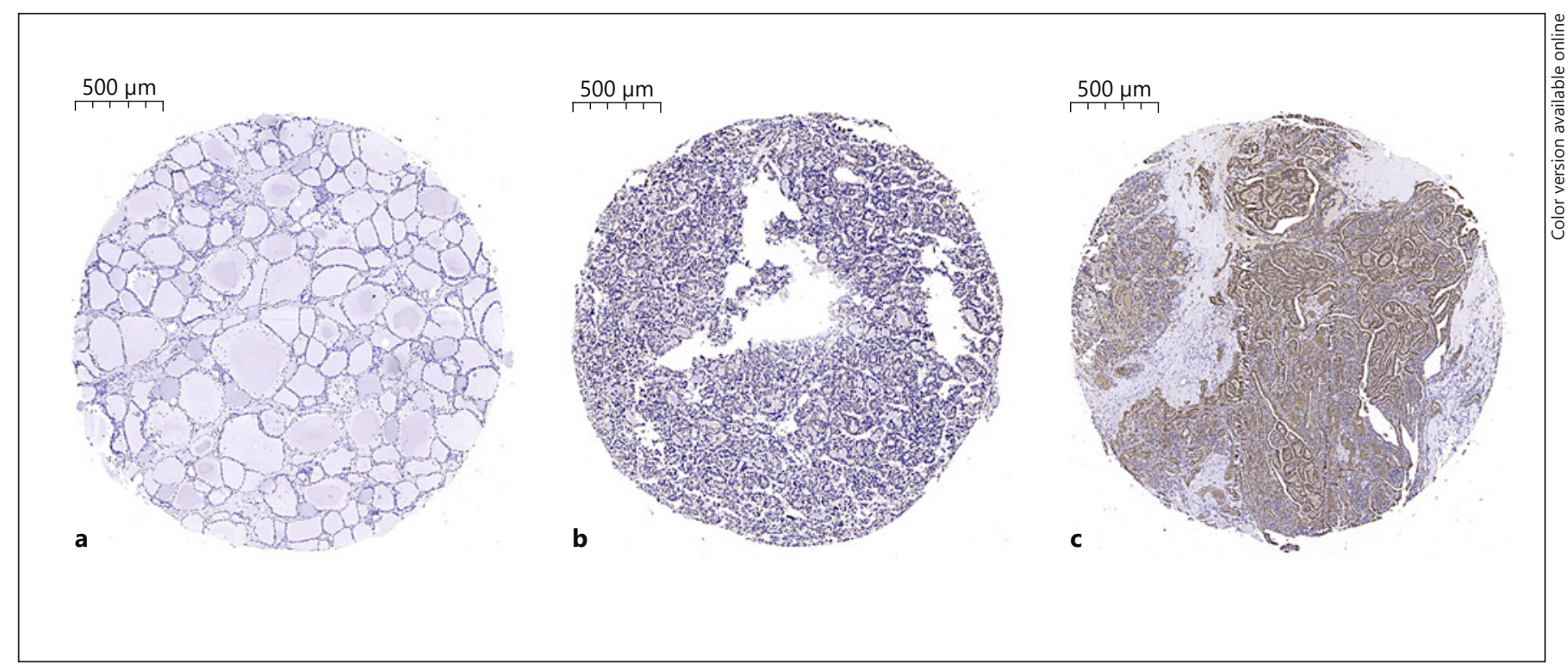

Fig. 2. Expression of IL-12A in normal thyroid tissues and thyroid cancer tissues detected by immunohistochemistry. a Expression of IL-12A in paracancerous tissues. b Low expression of IL-12A in thyroid cancer. c High expression of IL-12A in thyroid cancer. 
Table 2. Correlations of IL-12A expression with clinicopathological characteristics and outcomes of patients with differentiated thyroid cancer

\begin{tabular}{|c|c|c|c|c|c|}
\hline \multirow[t]{2}{*}{ Variables } & \multirow{2}{*}{$\begin{array}{l}\text { Total, } \\
n\end{array}$} & \multicolumn{2}{|c|}{ IL-12A expression, $n$} & \multirow[t]{2}{*}{$x^{2}$} & \multirow{2}{*}{$\begin{array}{l}p \\
\text { value }\end{array}$} \\
\hline & & low & high & & \\
\hline \multicolumn{6}{|l|}{ Age } \\
\hline$<55$ years & 77 & 60 & 17 & 1.057 & 0.389 \\
\hline$\geq 55$ years & 24 & 21 & 3 & & \\
\hline \multicolumn{6}{|l|}{ Sex } \\
\hline Male & 29 & 22 & 7 & 0.482 & 0.488 \\
\hline Female & 72 & 59 & 13 & & \\
\hline \multicolumn{6}{|l|}{ DTC } \\
\hline PTC & 87 & 72 & 15 & 2.591 & 0.145 \\
\hline FTC & 14 & 9 & 5 & & \\
\hline \multicolumn{6}{|l|}{ Multifocality } \\
\hline No & 70 & 56 & 14 & 0.006 & 0.940 \\
\hline Yes & 31 & 25 & 6 & & \\
\hline \multicolumn{6}{|l|}{ Tumor size } \\
\hline$<2 \mathrm{~cm}$ & 71 & 61 & 10 & 4.920 & 0.027 \\
\hline$\geq 2 \mathrm{~cm}$ & 30 & 20 & 10 & & \\
\hline \multicolumn{6}{|c|}{ Extrathyroid extension } \\
\hline No & 76 & 63 & 13 & 1.406 & 0.256 \\
\hline Yes & 25 & 18 & 7 & & \\
\hline \multicolumn{6}{|l|}{ T stage } \\
\hline $\mathrm{T} 1-\mathrm{T} 2$ & 67 & 56 & 11 & 1.435 & 0.231 \\
\hline T3-T4 & 34 & 25 & 9 & & \\
\hline \multicolumn{6}{|c|}{ Lymph node metastasis } \\
\hline No & 25 & 21 & 4 & 0.302 & 0.774 \\
\hline Yes & 76 & 60 & 16 & & \\
\hline \multicolumn{6}{|c|}{ Distant metastasis } \\
\hline No & 93 & 76 & 17 & 1.714 & 0.192 \\
\hline Yes & 8 & 5 & 3 & & \\
\hline \multicolumn{6}{|c|}{ Risk stratification } \\
\hline Low & 18 & 18 & 0 & 5.408 & 0.020 \\
\hline \multicolumn{6}{|c|}{ Intermediate } \\
\hline to high & 83 & 63 & 20 & & \\
\hline \multicolumn{6}{|l|}{ TNM stage } \\
\hline I-II & 97 & 80 & 17 & 7.991 & 0.024 \\
\hline III-IV & 4 & 1 & 3 & & \\
\hline \multicolumn{6}{|c|}{ Lymphocytic thyroiditis } \\
\hline No & 75 & 56 & 19 & 5.613 & 0.018 \\
\hline Yes & 26 & 25 & 1 & & \\
\hline \multicolumn{6}{|c|}{ Persistence or recurrence } \\
\hline No & 88 & 74 & 14 & 6.524 & 0.020 \\
\hline Yes & 13 & 7 & 6 & & \\
\hline
\end{tabular}

Bold typeface denotes significance. IL-12A, interleukin-12A; DTC, differentiated thyroid cancer; FTC, follicular thyroid cancer; PTC, papillary thyroid cancer; T stage, tumor stage; TNM stage, Tumor-Node-Metastasis stage.

mean age of $36.2 \pm 14.6$ years (range 19-65). A total of 81 patients were in the low IL-12A expression group, including 22 males and 59 females, with a mean age of $42.7 \pm$
Table 3. Multivariate logistic analysis of clinicopathological characteristics influencing IL-12A expression

\begin{tabular}{llll}
\hline Variables & OR & $95 \%$ CI & $p$ value \\
\hline $\begin{array}{l}\text { Tumor size } \\
\quad<2 \mathrm{~cm} \\
\quad 2 \mathrm{~cm}\end{array}$ & 1 & & \\
$\begin{array}{l}\text { Risk stratification } \\
\quad \text { Low }\end{array}$ & 2.315 & $0.760-7.054$ & 0.140 \\
$\quad$ Intermediate to high & 5.996 & $1.191-30.198$ & $\mathbf{0 . 0 3 0}$ \\
$\begin{array}{l}\text { TNM stage } \\
\quad \text { I-II }\end{array}$ & 1 & & \\
$\quad$ III-IV & 0.886 & $0.378-2.075$ & 0.780 \\
Lymphocytic thyroiditis & & & \\
$\quad$ No & 1 & & 0.061 \\
$\quad$ Yes & 0.132 & $0.016-1.095$ & 0.061 \\
\hline
\end{tabular}

Bold typeface denotes significance. IL-12A, interleukin-12A; TNM stage, Tumor-Node-Metastasis stage; OR, odds ratio; CI, confidence interval.

13.7 years (range 17-82). There was no significant difference in mean age between the two groups $(p=0.063)$. According to the American Joint Committee on Cancer TNM classification system (8th edition), the enrolled patients were divided into a young group ( $<55$ years) and an old group ( $\geq 55$ years). The association of the IL-12A expression level with clinicopathological features was first evaluated with a $\chi^{2}$ test (Table 2). No significant associations were found between IL-12A expression and age, sex, pathological type, and multifocality (all $p>0.05$; Table 2). IL-12A expression was negatively correlated with lymph node metastasis (lymphocytic thyroiditis [LT]; $p=0.018$ ); however, multivariate logistic regression analysis showed that LT was no independent predictor of IL-12A expression $(p=0.061$; Table 3$)$.

\section{Positive Association of IL-12A with Aggressiveness of DTC}

The relationship between clinicopathological signs of aggressiveness and IL-12A expression was analyzed, as shown in Table 2. Although IL-12A was not associated with extrathyroid extension, cervical lymph node metastasis, and distant metastasis, it was positively associated with tumor size $(p=0.027)$, risk stratification level $(p=0.020)$, and TNM stage $(p=0.024)$. Multivariate logistic regression analysis defined risk stratification as the independent factor to predict the expression of IL$12 \mathrm{~A}(p=0.030, \mathrm{OR}=5.996$ [95\% CI: 1.191-30.198]; Table 3$)$. Of the 101 patients, there were 13 (12.9\%) suffering from $\mathrm{P} \& \mathrm{R}$, of whom 2 patients were diagnosed with 
Table 4. Univariate analysis of significant prognostic factors influencing disease-free survival of 101 patients with differentiated thyroid cancer

\begin{tabular}{|c|c|c|c|c|}
\hline Variables & $\begin{array}{l}\text { Patients, } \\
n\end{array}$ & $\begin{array}{l}\mathrm{P} \& \mathrm{R}, \\
n\end{array}$ & $\begin{array}{l}\text { log-rank } \\
\text { value }\end{array}$ & $p$ value \\
\hline \multicolumn{5}{|l|}{ Age } \\
\hline$<55$ years & 77 & 9 & 0.458 & 0.499 \\
\hline$\geq 55$ years & 24 & 4 & & \\
\hline \multicolumn{5}{|l|}{ Sex } \\
\hline Male & 29 & 4 & 0.055 & 0.815 \\
\hline Female & 72 & 9 & & \\
\hline \multicolumn{5}{|c|}{ Pathology of DTC } \\
\hline FTC & 14 & 3 & 1.160 & 0.282 \\
\hline PTC & 87 & 10 & & \\
\hline \multicolumn{5}{|l|}{ Multifocality } \\
\hline No & 70 & 7 & 1.605 & 0.205 \\
\hline Yes & 31 & 6 & & \\
\hline \multicolumn{5}{|l|}{ Tumor size } \\
\hline$<2 \mathrm{~cm}$ & 71 & 5 & 7.511 & 0.006 \\
\hline$\geq 2 \mathrm{~cm}$ & 30 & 8 & & \\
\hline \multicolumn{5}{|c|}{ Extrathyroid extension } \\
\hline Yes & 76 & 7 & 3.904 & 0.048 \\
\hline No & 25 & 6 & & \\
\hline \multicolumn{5}{|l|}{ T stage } \\
\hline T1-T2 & 67 & 4 & 9.177 & 0.002 \\
\hline T3-T4 & 34 & 9 & & \\
\hline \multicolumn{5}{|c|}{ Lymph node metastasis } \\
\hline No & 25 & 2 & 0.695 & 0.404 \\
\hline Yes & 76 & 11 & & \\
\hline \multicolumn{5}{|l|}{ TNM stage } \\
\hline I-II & 97 & 11 & 7.984 & 0.005 \\
\hline III-IV & 4 & 2 & & \\
\hline \multicolumn{5}{|c|}{ Lymphocytic thyroiditis } \\
\hline No & 75 & 9 & 0.184 & 0.668 \\
\hline Yes & 26 & 4 & & \\
\hline \multicolumn{5}{|c|}{ IL-12A expression } \\
\hline Low & 81 & 7 & 6.323 & 0.012 \\
\hline High & 20 & 6 & & \\
\hline
\end{tabular}

Bold typeface denotes significance. IL-12A, interleukin-12A; DTC, differentiated thyroid cancer; FTC, follicular thyroid cancer; PTC, papillary thyroid cancer; background disease, Hashimoto thyroiditis or lymphocytic thyroiditis; T stage, tumor stage; TNM stage, Tumor-Node-Metastasis stage; $\mathrm{P} \& \mathrm{R}$, persistence or recurrence.

criterion 1 mentioned above (in subsection Criteria for P\&R of DTC), 3 patients with criterion 2, 3 patients with criterion 3 , and 5 patients with criterion 4 . The P\&R group consisted of 4 males and 9 females, with a mean age of $42.8 \pm 18.9$ years (range 24-82). A statistical test found that IL-12A expression was significantly higher in the $\mathrm{P} \& \mathrm{R}$ group than in the non- $\mathrm{P} \& \mathrm{R}$ group $(p=0.020$; Table 2).
High Expression of IL-12A Is an Independent

Predictor of Poor DFS of Patients with DTC

DFS was defined as the time from the date of surgery to the diagnosis of distant metastasis or recurrence or the date of the last follow-up. The mean follow-up time was $44.8 \pm 13.9$ months (range 0-60). Three patients died during follow-up; 1 patient died of thyroid cancer and 2 died of tumors in other organs. DFS was significantly different between the two groups ( $13.6 \pm 12.8$ vs. $49.4 \pm 5.7$ months, $p<0.001$ ), with shorter DFS in the $\mathrm{P} \& \mathrm{R}$ group than in the non-P\&R group.

The Kaplan-Meier method and log-rank test were applied to assess the impact of the clinicopathological factors and the rate of IL-12A positivity on P\&R of thyroid cancer. The results revealed that the rate of IL-12A positivity was associated with the DFS of DTC patients $(p=$ 0.012; Fig. 1c; Table 4). Moreover, factors such as tumor size, extrathyroid extension, T stage, and TNM stage also affected DFS, with $p$ values of $0.006,0.048,0.002$, and 0.005 , respectively. The details of the log-rank test and Kaplan-Meier curves are shown in Table 4 and Figure 3.

Multivariate Cox proportional-hazards analysis was performed to determine how strong the relationship was between IL-12A expression and the DFS of DTC patients. The results showed that there was still a strong association between tumor size, IL-12A expression, and DFS (all $p<0.05$ ), after excluding the effect of other factors (Table 5). The risk of $P \& R$ was more than 4 times higher among patients with tumor sizes $\geq 2 \mathrm{~cm}$ than among those with tumor sizes $<2 \mathrm{~cm}(p=0.031$, HR $=4.041$ [95\% CI: 1.14414.274]), and it was more than 4 times higher among patients with high IL-12A expression than among those with low IL-12A expression ( $p=0.049, \mathrm{HR}=4.027$ [95\% CI: 1.014-15.994]). Therefore, a tumor size $\geq 2 \mathrm{~cm}$ and high IL-12A expression are considered independent predictors of worse outcome in DTC patients.

\section{Discussion}

The data on 101 patients were retrospectively analyzed. We found that IL-12A was expressed in both normal thyroid tissues and DTC tissues, while the expression of IL-12A was significantly enhanced in DTC specimens. IL-12A expression in thyroid cancer was not associated with clinicopathological features such as age, gender, pathological type, multifocality, extrathyroid extension, lymph node metastasis, and distant metastasis. High IL$12 \mathrm{~A}$ expression was associated with aggressiveness of the DTC, as shown by the positive relationship of the IL-12A 


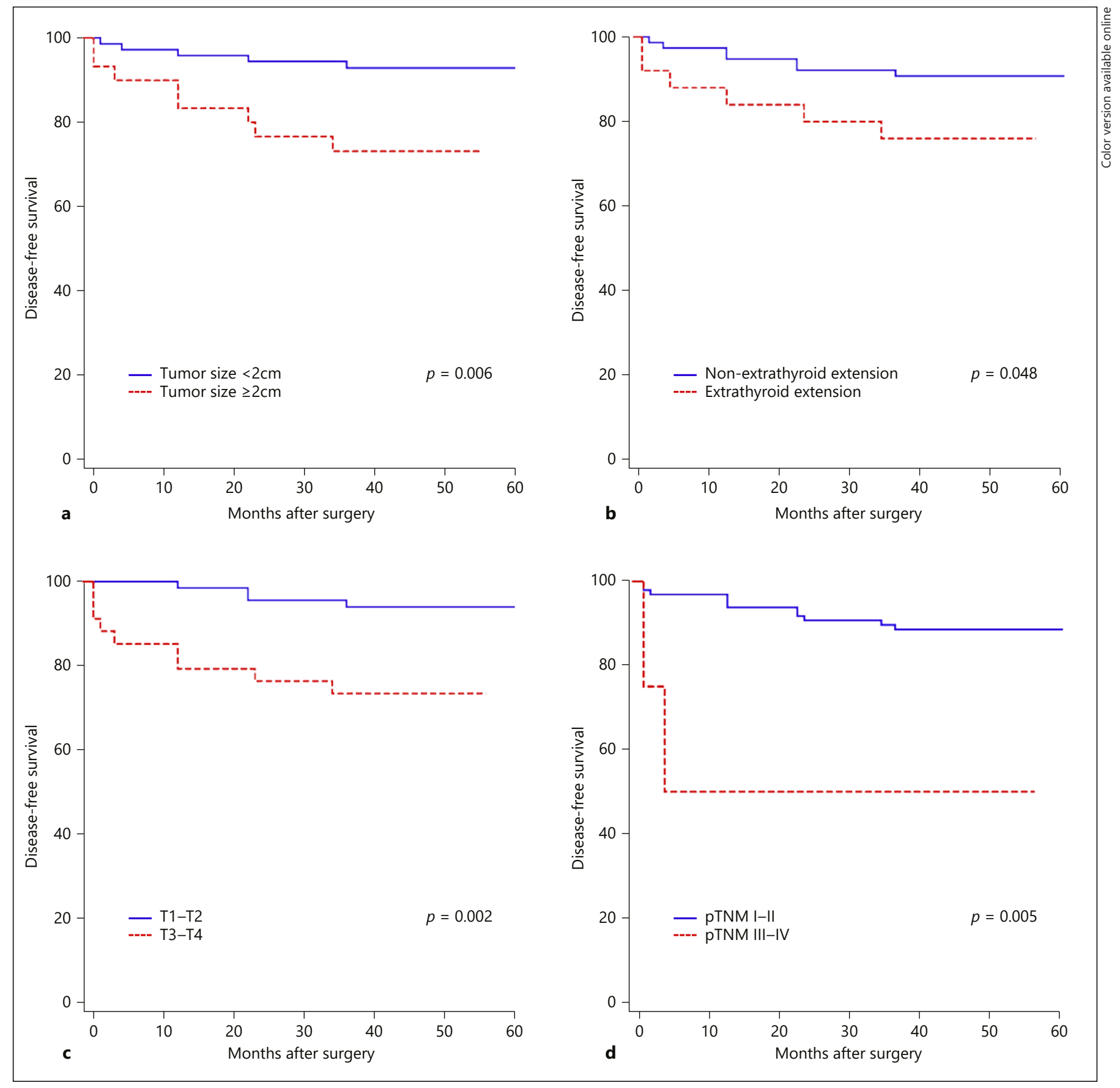

Fig. 3. Kaplan-Meier curves of disease-free survival according to tumor size (a), extrathyroid extension (b), T stage $(\mathbf{c})$, and pTNM stage $(\mathbf{d})$.

positivity rate to malignant indicators such as tumor size, risk stratification, and TNM stage. LT was found in 25.7\% of the DTC patients in the present study, which was lower than the rates in previous reports $[19,20]$. This difference may be due to the small sample size, selection, and the regional bias of our study. Multivariate logistic analy- sis defined risk stratification as an independent predictor of IL-12A expression. The frequency of P\&R of DTC was $12.9 \%$, which was similar to the rate of $11.4 \%$ that was reported by Trimboli et al. [21]. We showed that IL-12A was related to $\mathrm{P} \& \mathrm{R}$ of thyroid cancer. Furthermore, we demonstrated that the risk of $\mathrm{P} \& \mathrm{R}$ significantly increased 
Table 5. Multivariate analysis of prognostic factors for diseasefree-survival of 101 differentiated thyroid cancer patients using Cox's proportional-hazards model

\begin{tabular}{|c|c|c|c|}
\hline Variables & HR & $95 \% \mathrm{CI}$ & $p$ value \\
\hline \multicolumn{4}{|l|}{ Age } \\
\hline$<55$ years & 1 & & \\
\hline$\geq 55$ years & 3.335 & $0.811-13.720$ & 0.097 \\
\hline \multicolumn{4}{|l|}{ Sex } \\
\hline Male & 1 & & \\
\hline Female & 1.099 & $0.328-3.682$ & 0.879 \\
\hline \multicolumn{4}{|c|}{ Pathology of DTC } \\
\hline FTC & 1 & & \\
\hline PTC & 0.215 & $0.030-1.545$ & 0.129 \\
\hline \multicolumn{4}{|l|}{ Tumor size } \\
\hline$<2 \mathrm{~cm}$ & 1 & & \\
\hline$\geq 2 \mathrm{~cm}$ & 4.041 & $1.144-14.274$ & 0.031 \\
\hline \multicolumn{4}{|l|}{ Multifocality } \\
\hline No & 1 & & \\
\hline Yes & 2.450 & $0.688-8.725$ & 0.169 \\
\hline \multicolumn{4}{|c|}{ Extrathyroid extension } \\
\hline No & 1 & & \\
\hline Yes & 1.634 & $0.424-6.298$ & 0.478 \\
\hline \multicolumn{4}{|c|}{ Lymph node metastasis } \\
\hline No & 1 & & \\
\hline Yes & 5.858 & $0.820-41.834$ & 0.080 \\
\hline \multicolumn{4}{|c|}{ Lymphocytic thyroiditis } \\
\hline No & 1 & & \\
\hline Yes & 4.007 & $0.842-19.058$ & 0.083 \\
\hline \multicolumn{4}{|c|}{ IL-12A expression } \\
\hline Low & 1 & & \\
\hline High & 4.027 & $1.014-15.994$ & 0.049 \\
\hline
\end{tabular}

Bold typeface denotes significance. IL-12A, interleukin-12A; DTC, differentiated thyroid cancer; FTC, follicular thyroid cancer; PTC, papillary thyroid cancer; $\mathrm{HR}$, hazard ratio; CI, confidence interval.

in patients with high IL-12A expression, and IL-12A was an independent factor for predicting poor prognosis in patients with DTC.

The IL-12 family consists of a group of cytokines that have different impacts on the outcome of cancer, infection, and inflammatory diseases [10]. Chain sharing occurs extensively in this heterodimeric cytokine family and its receptors. All cytokines of the IL-12 family are composed of an $\alpha$-chain and a $\beta$-chain, which are connected by a disulfide bond. The $\alpha$-chain includes three components (P35, P19, and P28) and the $\beta$-chain includes two components (P40 and Ebi3). The P40 chain pairs with P35 or P19 to form IL-12 or IL-23, while Ebi3 pairs with P28 or P35 to form IL-27 or IL-35, respectively. The a-chain P35 (IL-12A) is a constituent of both IL-12 and
IL-35, which are two cytokines that have antagonistic effects on the tumor [16].

IL-12 was reported to be more highly expressed in tumor specimens than in the normal tissues of corresponding organs. In addition, it was well recognized as a cytokine that acts against tumors, with high expression predicting a superior outcome in cancer patients [22, 23]. However, the present study found that IL-12A was highly expressed in DTC and was associated with poor outcome. Part of the reason for the contradictory results is that IL$12 \mathrm{~A}$ expression in this study simultaneously reflected the total expression of both IL-12 and IL-35, because IL-12A is a component of both of these cytokines. Whereas the expression of IL-12 was decreased in tumor tissues, the expression of IL-35 was significantly upregulated. Therefore, the high expression of IL-12A was mainly the result of IL-35 expression. Although the heterodimeric structure is a key feature of IL-12 family members, studies have reported that the subunits of this family can exist and function as monomers and homodimers. The results of this study suggest that IL-12A may exist in the tumor microenvironment as a monomer and as a homodimer, which competitively inhibit the effect of IL-12 or function as negative regulatory cytokines of immune responses against tumors. The exact structural forms, functions, and mechanisms of IL-12A in the DTC microenvironment need to be further explored in future studies.

In conclusion, the present study demonstrates that IL$12 \mathrm{~A}$ is highly expressed in DTC. In addition, the IL-12A expression level is associated with the aggressiveness of the disease. Moreover, IL-12A could act as an independent predictive factor for the outcome of DTC.

\section{Acknowledgements}

We thank Asia-Vector Biotechnology Co. Ltd (Shanghai, China) for making the tissue microarray.

\section{Statement of Ethics}

The study protocol was approved by the Ethics Committee of Shanghai Jiao Tong University Affiliated Sixth People's Hospital. Written informed consent was obtained from the 101 enrolled patients.

\section{Disclosure Statement}

There is no conflict of interest to declare. 


\section{Funding Sources}

This study was supported by the National Natural Science Foundation of China, under grant No. 81771865, and by the Shanghai Key Discipline of Medical Imaging, under grant No. $2017 Z Z 02005$.

\section{Author Contributions}

The study was designed by Q.-Y. Luo and Z.-L. Qiu; H.-J. Song collected the pathological tissue samples; G.-Q. Zhang and C.-T. Shen conducted the statistical analysis and wrote the paper.

\section{References}

1 Huang C, Yang X, Han L, Fan Z, Liu B, Zhang $\mathrm{C}$, et al. The prognostic potential of alpha- 1 type I collagen expression in papillary thyroid cancer. Biochem Biophys Res Commun. 2019 Jul;515(1):125-32.

2 Karagiannis AK, Philippou A, Tseleni-Balafouta S, Zevolis E, Nakouti T, Tsopanomichalou-Gklotsou M, et al. IGF-IEc Expression Is Associated with Advanced Differentiated Thyroid Cancer. Anticancer Res. 2019 Jun; 39(6):2811-9.

3 Haugen BR, Alexander EK, Bible KC, Doherty GM, Mandel SJ, Nikiforov YE, et al. 2015 American Thyroid Association Management Guidelines for Adult Patients with Thyroid Nodules and Differentiated Thyroid Cancer: The American Thyroid Association Guidelines Task Force on Thyroid Nodules and Differentiated Thyroid Cancer. Thyroid. 2016; 26(1):1-133.

4 Cunha LL, Morari EC, Nonogaki S, Marcello MA, Soares FA, Vassallo J, et al. Interleukin 10 expression is related to aggressiveness and poor prognosis of patients with thyroid cancer. Cancer Immunol Immunother. $2017 \mathrm{Feb}$; 66(2):141-8.

5 Balkwill F, Mantovani A. Inflammation and cancer: back to Virchow? Lancet. 2001 Feb; 357(9255):539-45.

6 Zhao Y, Dong Q, Li J, Zhang K, Qin J, Zhao J, et al. Targeting cancer stem cells and their niche: perspectives for future therapeutic targets and strategies. Semin Cancer Biol. 2018 Dec;53:139-55.

7 Cunha LL, Marcello MA, Ward LS. The role of the inflammatory microenvironment in thyroid carcinogenesis. Endocr Relat Cancer. 2014 Apr;21(3):R85-103.
8 Fallahi P, Ferrari SM, Piaggi S, Luconi M, Cantini G, Gelmini S, et al. The paramount role of cytokines and chemokines in papillary thyroid cancer: a review and experimental results. Immunol Res. 2018 Dec;66(6):710-22.

9 Akdis M, Burgler S, Crameri R, Eiwegger T, Fujita H, Gomez E, et al. Interleukins, from 1 to 37 , and interferon- $\gamma$ : receptors, functions, and roles in diseases. J Allergy Clin Immunol. 2011;127(3):701-21.e1-70.

10 Tait Wojno ED, Hunter CA, Stumhofer IS. The Immunobiology of the Interleukin-12 Family: Room for Discovery. Immunity. 2019 Apr;50(4):851-70.

11 Yuzhalin AE, Kutikhin AG. Interleukin-12: clinical usage and molecular markers of cancer susceptibility. Growth Factors. 2012 Jun; 30(3):176-91.

12 Han SS, Cho EY, Lee TS, Kim JW, Park NH, Song YS, et al. Interleukin-12 p40 gene (IL12B) polymorphisms and the risk of cervical cancer in Korean women. Eur J Obstet Gynecol Reprod Biol. 2008 Sep;140(1):71-5.

13 Navaglia F, Basso D, Zambon CF, Ponzano E, Caenazzo L, Gallo N, et al. Interleukin 12 gene polymorphisms enhance gastric cancer risk in $\mathrm{H}$ pylori infected individuals. J Med Genet. 2005 Jun;42(6):503-10.

14 Wu MF, Wang YC, Li HT, Chen WC, Liao $\mathrm{CH}$, Shih TC, et al. The Contribution of Interleukin-12 Genetic Variations to Taiwanese Lung Cancer. Anticancer Res. 2018 Nov; 38(11):6321-7.

15 Martins MB, Marcello MA, Batista FA, Peres KC, Meneghetti M, Ward MA, et al. Serum interleukin measurement may help identify thyroid cancer patients with active disease. Clin Biochem. 2018 Feb;52:1-7.

16 Vignali DA, Kuchroo VK. IL-12 family cytokines: immunological playmakers. Nat Immunol. 2012 Jul;13(8):722-8.
17 Rosenbaum MW, Gigliotti BJ, Pai SI, Parangi $\mathrm{S}$, Wachtel H, Mino-Kenudson M, et al. PDL1 and IDO1 Are Expressed in Poorly Differentiated Thyroid Carcinoma. Endocr Pathol. 2018 Mar;29(1):59-67.

18 Camp RL, Dolled-Filhart M, Rimm DL. Xtile: a new bio-informatics tool for biomarker assessment and outcome-based cut-point optimization. Clin Cancer Res. 2004;10(21): 7252-9.

19 Borowczyk M, Janicki A, Dworacki G, Szczepanek-Parulska E, Danieluk M, Barnett J, et al. Decreased staging of differentiated thyroid cancer in patients with chronic lymphocytic thyroiditis. J Endocrinol Invest. 2019 Jan; 42(1):45-52.

20 Donangelo I, Walts AE, Bresee C, Braunstein GD. Lymphocytic thyroiditis is associated with increased number of benign cervical nodes and fewer central neck compartment metastatic lymph nodes in patients with differentiated thyroid cancer. Endocr Pract. 2016 Oct;22(10):1192-8.

21 Trimboli P, Zilioli V, Imperiali M, Giovanella L. Thyroglobulin autoantibodies before radioiodine ablation predict differentiated thyroid cancer outcome. Clin Chem Lab Med. 2017 Oct;55(12):1995-2001.

22 Bien E, Krawczyk M, Izycka-Swieszewska E, Trzonkowski P, Kazanowska B, Adamkiewicz-Drozynska E, et al. Deregulated systemic IL-10/IL-12 balance in advanced and poor prognosis paediatric soft tissue sarcomas. Biomarkers. 2013;18(3):204-15.

23 Youssef SS, Mohammad MM, Ezz-El-Arab LR. Clinical Significance of Serum IL-12 Level in Patients with Early Breast Carcinoma and Its Correlation with Other Tumor Markers. Open Access Maced J Med Sci. 2015 Dec; 3(4):640-4. 